Received 09/25/2021 Review began 10/04/2021 Review ended 10/21/2021 Published 10/25/2021

\section{(c) Copyright 2021}

Chen et al. This is an open access article distributed under the terms of the Creative Commons Attribution License CC-BY 4.0. which permits unrestricted use, distribution, and reproduction in any medium, provided the original author and source are credited.

\title{
Superior Ophthalmic Vein Thrombosis Induced by Orbital Cellulitis: An Ophthalmic Emergency
}

\author{
Lu Chen ${ }^{1}$, Uta S. Guo ${ }^{2}$, Gennadiy Grutman ${ }^{3}$, Samy I. McFarlane ${ }^{4}$, Parag Mehta ${ }^{3}$ \\ 1. Department of Cardiology, State University of New York Downstate Medical Center, Brooklyn, USA 2. Department of \\ Family Medicine and Community Health, Icahn School of Medicine at Mount Sinai, New York, USA 3. Internal \\ Medicine, New York-Presbyterian Brooklyn Methodist Hospital, Brooklyn, USA 4. Department of Medicine and \\ Endocrinology, State University of New York Downstate Medical Center, Brooklyn, USA
}

Corresponding author: Lu Chen, lu.chen@downstate.edu

\begin{abstract}
Superior ophthalmic vein thrombosis (SOVT) is a rare ophthalmologic emergency. The most common etiologies include infection, trauma, inflammation, and malignancy, as well as thyroid-related orbitopathy. Early identification and timely intervention can lead to a significant decrease in complications that include cavernous sinus thrombosis (CST), vision loss, and death. This rare disease entity almost always makes its initial presentation to internal medicine or emergency medicine (EM) physicians. In this report, we present a case of SOVT that presented overnight to the emergency department for worsening right facial swelling and orbital pain. We discuss our experience with the evaluation and management of SOVT and provide a review of the currently available literature to emphasize the importance of obtaining a full history and physical examination, seeking early imaging studies, and ophthalmology consultation for patients with suspected SOVT.
\end{abstract}

Categories: Internal Medicine, Medical Education, Ophthalmology

Keywords: bacterial infection, cavernous sinus thrombosis, ophthalmologic emergency, orbital cellulitis, superior ophthalmic vein thrombosis

\section{Introduction}

Primary care and emergency medicine (EM) physicians serve as the initial medical contact for patients with a broad spectrum of diseases. Superior ophthalmic vein thrombosis (SOVT) is a rare ophthalmologic emergency almost always first presented in the primary care or emergency setting. The presentation of SOVT varies depending on the underlying pathology, with the most common etiologies being infection, trauma, neoplasm, and vasculitis [1]. The most commonly reported cases of SOVT are associated with infectious etiology of surrounding structures, such as orbital cellulitis (OC) [2-8], sinusitis [9,10], dental abscess [7,11], and cutaneous infection [12-14]. Due to the strong association with orbital cellulitis, suspicion of SOVT is high if the physical examination is suggestive of optic nerve or orbital involvement [1]. Reported complications include permanent impairment or loss of vision $[5,7,8,15]$ and death $[4,11,14]$.

We describe a case of OC-induced SOVT initially treated as conjunctivitis and, after further investigation, successfully managed with intravenous (IV) antibiotics and anticoagulation. The purpose of this case report is to emphasize the importance of obtaining a full history with a focused physical examination, seeking early imaging studies, and ophthalmology consultation for patients with suspected SOVT.

\section{Case Presentation}

A 44-year-old male with uncontrolled diabetes mellitus (DM) type II secondary to medication noncompliance presented overnight to the emergency department for worsening right hemifacial swelling and sharp orbital pain with flu-like symptoms that started 10 days prior. He reported right-sided ocular pain but denied changes in visual acuity or color perception. Physical examination revealed periorbital swelling, tenderness, and erythema that extended down to the right maxilla. Phlebitis was appreciable along the bilateral frontal sinuses and extended inferiorly to the nasal ridge. The ocular examination revealed chemosis, ptosis, and conjunctival injection in the right eye. The patient was admitted to medical service and started on acyclovir and clindamycin for suspected herpes zoster-induced conjunctivitis with possible sinusitis.

Despite aggressive management, reoccurring febrile episodes were noted overnight, and cellulitis appeared to worsen the following morning. Further history revealed that the patient works in construction and frequently wipes his face with gloved hands. He also reported medication noncompliance due to a lack of insurance. A thorough examination revealed ocular pain and pain with extraocular movement, with no diplopia. Visual acuity, color perception, and pupillary light reflex were bilaterally intact. Laboratory studies were remarkable for leukocytosis of $14,600 / \mathrm{mL}$ with neutrophil predominance and glycohemoglobin of $11.1 \%$. The remaining laboratory studies, including renal function and hepatic function, were unremarkable. 


\section{Cureus}

Maxillofacial computed tomography (CT) with IV contrast was obtained due to the concern of orbital involvement (Figures 1 and 2). CT scan demonstrated proptosis of the right eye, thrombosis of the right superior ophthalmic vein, and possible cavernous sinus thrombosis (CST). Orbital cellulitis was suspected, and anticoagulation with unfractionated heparin was initiated. Ophthalmology consultation was requested, and the patient was transferred to the intensive care unit in a tertiary care center. The patient was managed conservatively and discharged home on oral antibiotics with outpatient follow-up. At three-month followup, the patient reported complete resolution of symptoms with no impairment of visual acuity. He also reported medication compliance at this time.

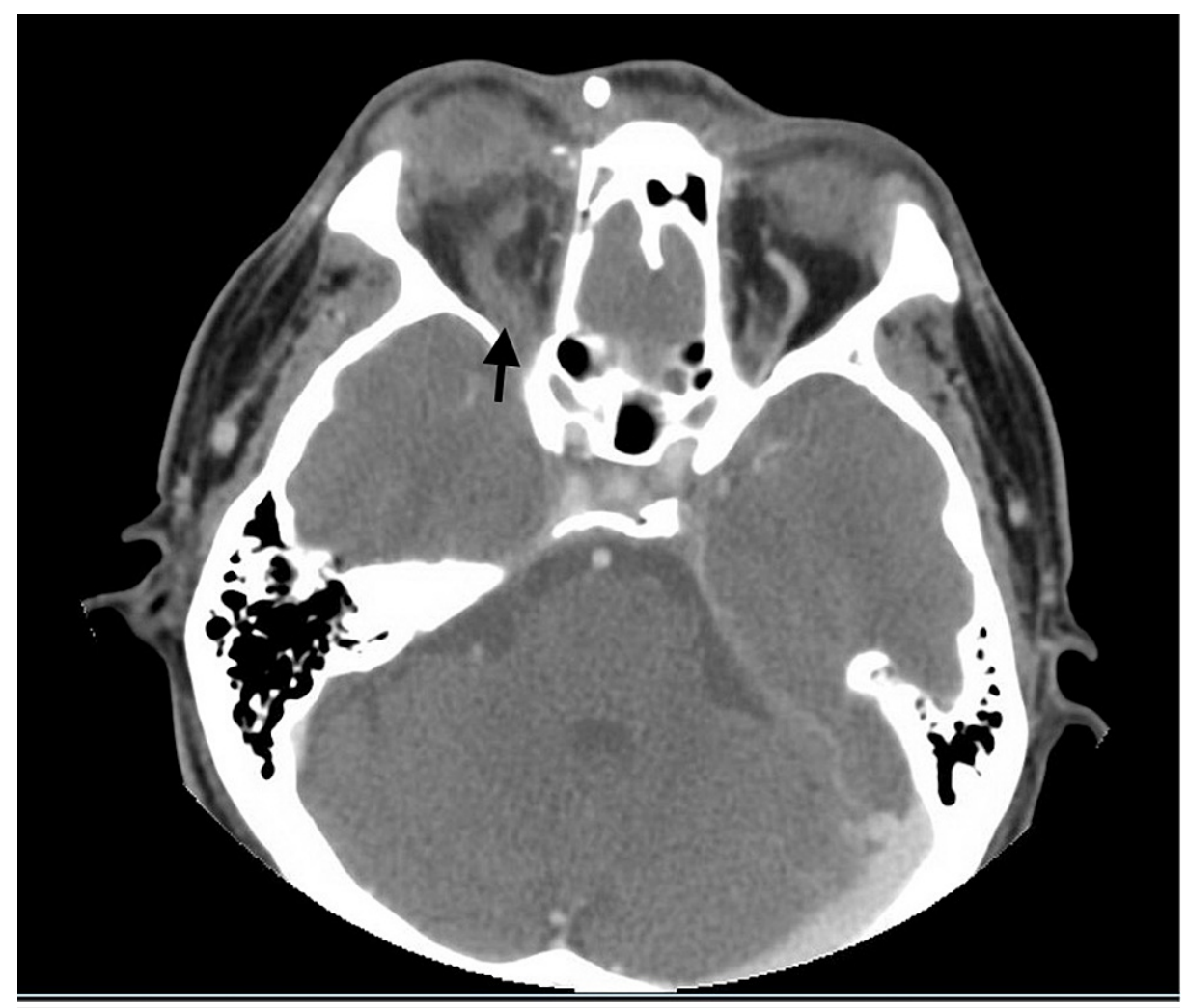

FIGURE 1: Right-sided superior ophthalmic vein thrombosis. Enlargement of the superior ophthalmic vein and lack of contract uptake (arrow). 


\section{Cureus}

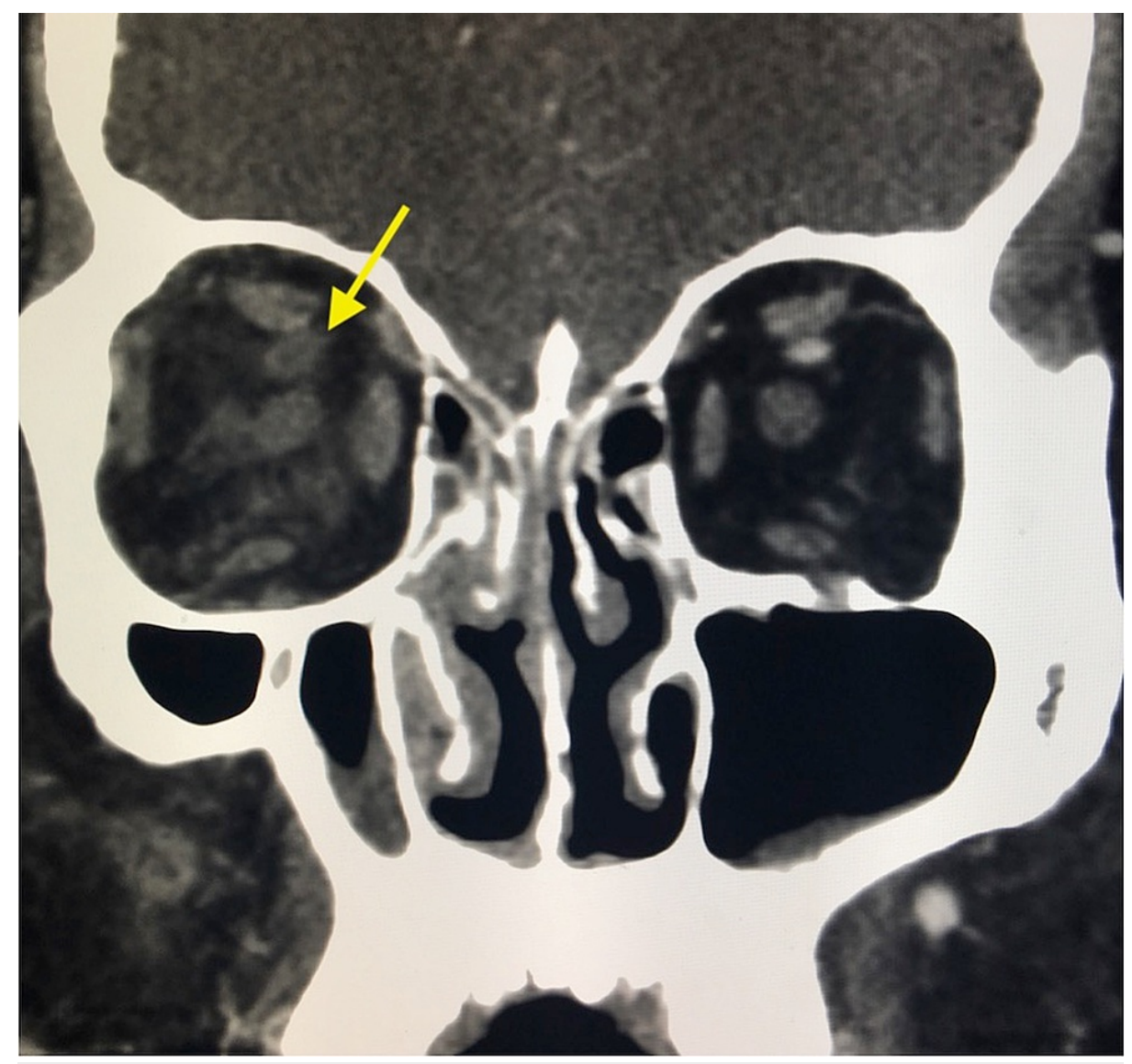

FIGURE 2: Coronal view of the right-sided superior ophthalmic vein thrombosis (arrow), with fat stranding suggestive of congestive edema and inflammation.

\section{Discussion}

SOVT is a rare but serious clinical condition that requires immediate intervention. Delay in care can result in permanent blindness and fatality. Infection-induced SOVT usually presents with orbital cellulitis, frequently as a result of direct inoculation from sinusitis [1]. Due to the valveless nature of the superior ophthalmic veins and facial veins, a seemingly innocuous superficial infection in the danger triangle can spread posteriorly to the cavernous sinus leading to a CST or meningitis [16]. In our patient with a relative immunocompromised state from uncontrolled DM, frequent face wiping at the construction site became the likely nidus of infection.

The distinguishing feature of SOVT is optic nerve or orbital involvement. The impairment of visual acuity, color perception, and pupillary light reflex can suggest optic neuropathy. In addition, pain or paralysis with eye movements, proptosis, or diplopia suggests inflammation of extraocular muscles and thus orbital involvement $[17,18]$. In the absence of symptoms of optic neuropathy and orbital cellulitis, superficial infections such as preseptal cellulitis are more likely. Based on available literature detailed in Table 1, SOVT can present in a wide heterogeneity in age, sex, comorbidities, and inciting infection. However, every patient diagnosed with infectious SOVT had more than one symptom or sign of optic nerve or orbital involvement (Table 1). The majority of patients present with unilateral ocular complaints, although bilateral ocular involvement has been reported as well [19]. Suspicion of either optic nerve or orbital involvement should prompt immediate imaging studies and urgent ophthalmology consultation to rule out SOVT and CST [18].

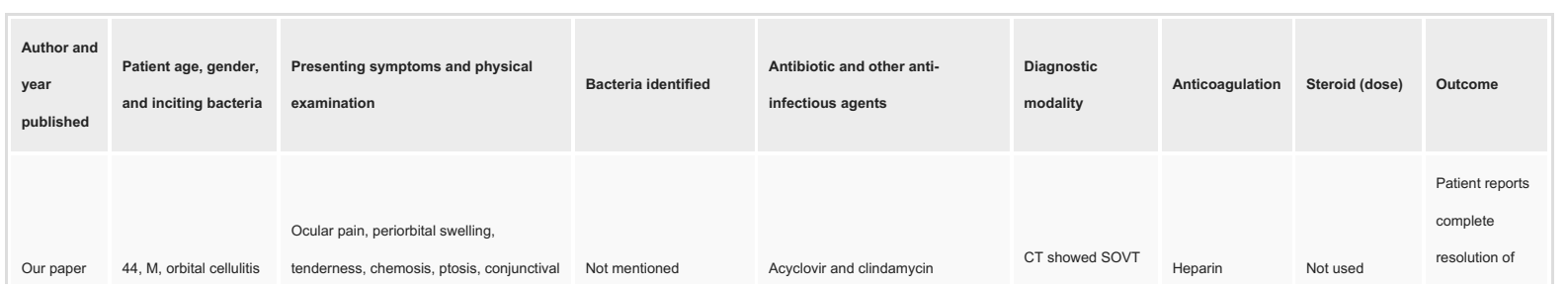




\section{Cureus}

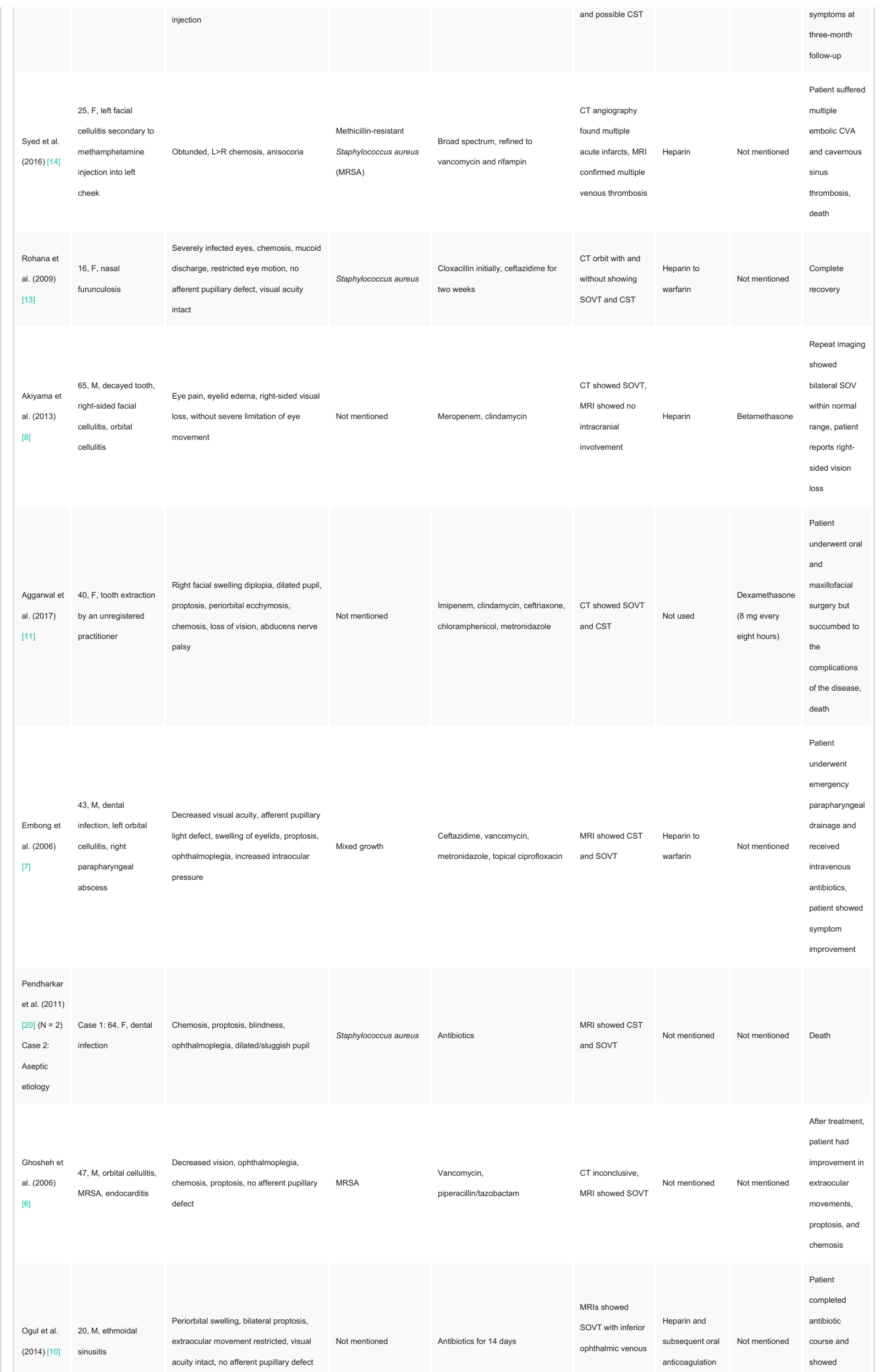




\section{Cureus}

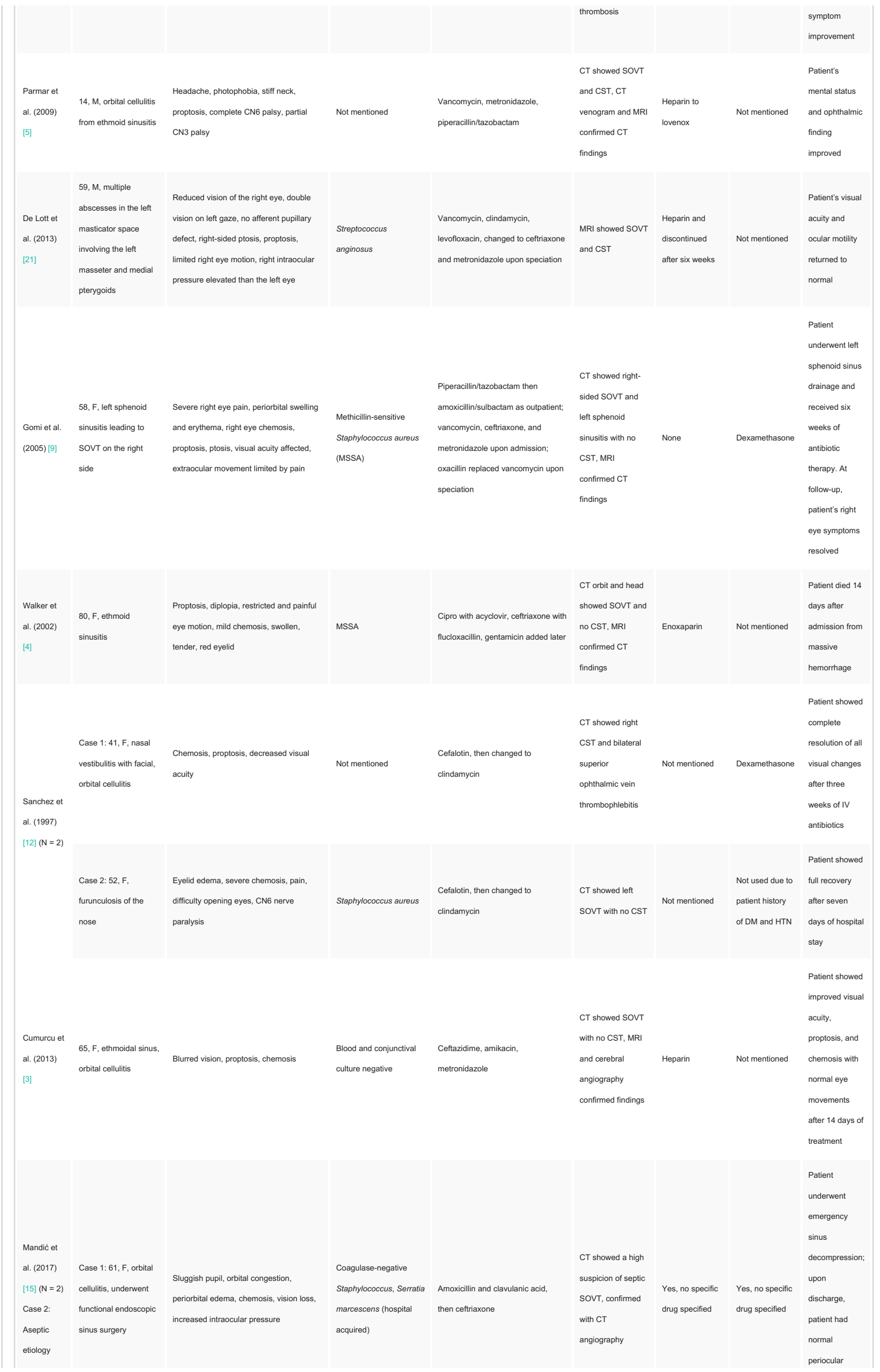




\section{Cureus}

TABLE 1: Review of available publications on superior ophthalmic vein thrombosis, including presentation, inciting bacteria, antibiotics used, and clinical outcome.

In patients with infectious SOVT, the most common bacteria was Staphylococcus aureus (seven out of 10 cases) [4,6,9,12-14,20], including two cases of methicillin-resistant Staphylococcus aureus (MRSA) [6,14]. Coagulation-negative Staphylococcus and Streptococcus anginosus were also reported [15,21]. Therefore, the initial choice of antibiotic should be broad spectrum, with coverage for Staphylococcus aureus. Coverage for MRSA should be individualized; previously reported cases of MRSA infection were both associated with the intravenous use of illicit drugs [6,14].

Initial anticoagulation and steroid use have been reported, although the benefit of either therapy is unclear at this time [1]. Anticoagulation therapy was used in 11 cases and considered in one additional case. The initial agents of anticoagulation used were heparin or low-molecular-weight heparin. Steroid therapy, most commonly dexamethasone, was used in five cases. The paucity of SOVT cases makes it difficult to standardize the use of anticoagulation of steroid therapy. The decision of either therapy should be individualized to a specific patient.

\section{Conclusions}

SOVT is a rare medical emergency often initially presented in the primary care or emergency care setting. A focused history and physical examination to rule out orbital involvement and optic neuropathy are critical. Early detection and treatment of SOVT prevent progression to CST and its detrimental sequelae. Fortunately, the majority of the cases reported complete recovery. Suspected cases of SOVT should trigger early imaging studies and urgent ophthalmology consultation to minimize detrimental complications.

\section{Additional Information \\ Disclosures}

Human subjects: Consent was obtained or waived by all participants in this study. New York Presbyterian Brooklyn Methodist Hospital Institutional Review Board issued approval n/a. This study was reviewed and exempted by the internal review board at our hospital. Conflicts of interest: In compliance with the ICMJE uniform disclosure form, all authors declare the following: Payment/services info: All authors have declared that no financial support was received from any organization for the submitted work. Financial relationships: All authors have declared that they have no financial relationships at present or within the previous three years with any organizations that might have an interest in the submitted work. Other relationships: All authors have declared that there are no other relationships or activities that could appear to have influenced the submitted work.

\section{Acknowledgements}

The authors would like to acknowledge Dr. Garen M Polatoglu for his dedication to the care of this patient.

\section{References}

1. Kumar JB, Colón-Acevedo B, Liss J, Fekrat S, Scott I: Diagnosis and management of superior ophthalmic vein thrombosis. Fekrat S, Scott IU (ed): EyeNet Magazine, London, England, United Kingdom; 2015.

2. Schmitt NJ, Beatty RL, Kennerdell JS: Superior ophthalmic vein thrombosis in a patient with dacryocystitisinduced orbital cellulitis. Ophthalmic Plast Reconstr Surg. 2005, 21:387-9. 10.1097/01.iop.0000176269.84949.96

3. Cumurcu T, Demirel S, Keser S, Bulut T, Cavdar M, Doğan M, Saraç K: Superior ophthalmic vein thrombosis developed after orbital cellulitis. Semin Ophthalmol. 2013, 28:58-60. 10.3109/08820538.2012.736007

4. Walker JC, Sandhu A, Pietris G: Septic superior ophthalmic vein thrombosis. Clin Exp Ophthalmol. 2002, 30:144-6. 10.1046/j.1442-6404.2002.00501.x

5. Parmar H, Gandhi D, Mukherji SK, Trobe JD: Restricted diffusion in the superior ophthalmic vein and cavernous sinus in a case of cavernous sinus thrombosis. J Neuroophthalmol. 2009, 29:16-20. 10.1097/WNO.0b013e31818e3f40

6. Ghosheh FR, Kathuria SS: Intraorbital heroin injection resulting in orbital cellulitis and superior ophthalmic vein thrombosis. Ophthalmic Plast Reconstr Surg. 2006, 22:473-5. 10.1097/01.iop.0000248991.71690.eb

7. Embong Z, Ismail S, Thanaraj A, Hussein A: Dental infection presenting with ipsilateral parapharyngeal abscess and contralateral orbital cellulitis - a case report. Malays J Med Sci. 2007, 14:62-6.

8. Akiyama K, Karaki M, Samukawa Y, Mori N: Blindness caused by septic superior ophthalmic vein thrombosis in a Lemierre Syndrome variant. Auris Nasus Larynx. 2013, 40:493-6. 10.1016/j.anl.2012.09.006

9. Gomi H, Gandotra SD, Todd CY: Septic right superior ophthalmic vein thrombosis complicated by left 
sphenoid sinusitis. Scand J Infect Dis. 2005, 36:316-8. 10.1080/00365540410021135-1

10. Ogul H, Gedikli Y, Karaca L, Okur A, Kantarci M: Massive thrombosis of bilateral superior and inferior ophthalmic veins secondary to ethmoidal rhinosunisitis: imaging findings. J Craniofac Surg. 2014, 25:e2779. 10.1097/SCS.0000000000000661

11. Aggarwal K, Rastogi S, Joshi A, Kumar A, Chaurasia A, Prakash R: Cavernous sinus thrombosis following dental extraction: a rare case report and forgotten entity. J Korean Assoc Oral Maxillofac Surg. 2017, 43:3515. 10.5125/jkaoms.2017.43.5.351

12. Sanchez TG, Cahali MB, Murakami MS, Butugan O, Miniti A: Septic thrombosis of orbital vessels due to cutaneous nasal infection. Am J Rhinol. 1997, 11:429-33. 10.2500/105065897780914947

13. Rohana AR, Rosli MK, Nik Rizal NY, Shatriah I, Wan Hazabbah WH: Bilateral ophthalmic vein thrombosis secondary to nasal furunculosis. Orbit. 2008, 27:215-7. 10.1080/01676830802009754

14. Syed A, Bell B, Hise J, Philip J, Spak C, Opatowsky MJ: Bilateral cavernous sinus and superior ophthalmic vein thrombosis in the setting of facial cellulitis. Proc (Bayl Univ Med Cent). 2016, 29:36-8. 10.1080/08998280.2016.11929349

15. Mandić JJ, Mandić K, Mrazovac D: Superior ophthalmic vein thrombosis with complete loss of vision as a complication of autoimmune and infective conditions. Ocul Immunol Inflamm. 2018, 26:1066-8. 10.1080/09273948.2017.1313433

16. Morton DA, Foreman K, Albertine KH: Superficial Face. The Big Picture: Gross Anatomy, 2e. McGraw Hill, New York, United States; 2019. 27:

17. Botting AM, McIntosh D, Mahadevan M: Paediatric pre- and post-septal peri-orbital infections are different diseases. a retrospective review of 262 cases. Int J Pediatr Otorhinolaryngol. 2008, 72:377-83. 10.1016/j.ijporl.2007.11.013

18. Rudloe TF, Harper MB, Prabhu SP, Rahbar R, Vanderveen D, Kimia AA: Acute periorbital infections: who needs emergent imaging?. Pediatrics. 2010, 125:e719-26. 10.1542/peds.2009-1709

19. van der Poel NA, de Witt KD, van den Berg R, de Win MM, Mourits MP: Impact of superior ophthalmic vein thrombosis: a case series and literature review. Orbit. 2019, 38:226-32. 10.1080/01676830.2018.1497068

20. Pendharkar HS, Gupta AK, Bodhey N, Nair M: Diffusion restriction in thrombosed superior ophthalmic veins: two cases of diverse etiology and literature review. J Radiol Case Rep. 2011, 5:8-16. 10.3941/jrcr.v5i3.547

21. De Lott LB, Trobe JD, Parmar H: Restricted diffusion of the superior and inferior ophthalmic veins in cavernous sinus thrombosis. J Neuroophthalmol. 2013, 33:268-70. 10.1097/01.wno.0000434279.57700.3c 